\title{
TAGUNG
}

\section{Erste Jahrestagung des Deutsch-Portugiesischen Forums: Perspektiven einer starken Partnerschaft in schweren Zeiten}

\author{
Ann-Sophie Gast*
}

Am 24. und 25. Januar 2013 fand die erste Jahrestagung des Deutsch-Portugiesischen Forums in der Stiftung Fundação Calouste Gulbenkian in Lissabon statt. Höhepunkt des Forums mit 300 Teilnehmern waren die Ansprachen des deutschen und des portugiesischen Außenministers. Sie wurden ergänzt durch Reden, thematische Panels und Diskussionsrunden, in denen hochrangige Vertreter aus Politik, Wirtschaft und Gesellschaft, Entscheidungsträger wie Akademiker über die deutschportugiesischen Beziehungen im europäischen und globalen Kontext debattierten.

Das Deutsch-Portugiesische Forum ist eine zivilgesellschaftliche Initiative, die vom Instituto Português de Relações Internacionais (IPRIUNL), der Fundação Calouste Gulbenkian in Lissabon und dem Institut für Europäische Politik (IEP) in Berlin koordiniert und getragen wird. Das bilaterale Forum steht allen gesellschaftlichen Gruppen offen und soll als wichtige Institution des gesellschaftlichen Dialogs zwischen Portugal und Deutschland den Gedanken- und Erfahrungsaustausch sowie die Vernetzung zwischen Experten aus Politik, Wirtschaft, Kultur, Wissenschaft und Zivilgesellschaft fördern.

Thema der ersten Jahrestagung des DeutschPortugiesischen Forums war die Schulden- und Vertrauenskrise in Europa. Es wurden nicht nur die Schwierigkeiten der Krisenbewältigung und die Bereiche, in denen akuter Handlungsbedarf besteht, identifiziert, sondern auch länderspezifische Lösungsmodelle und gemeinsame Antworten auf die Krise diskutiert.

\section{Erste Jahrestagung des Deutsch- Portugiesischen Forums}

Gemeinsames bilaterales Forum des Instituto Português de Relações Internacionais (IPRI-UNL), der Fundação Calouste Gulbenkian Lissabon und des Instituts für Europäische Politik (IEP) e.V. Berlin. Mit freundlicher Unterstützung des Auswärtigen Amtes, der Botschaft der Bundesrepublik Deutschland in Portugal, der portugiesischen Nachrichtenagentur Lusa und dem Goethe-Institut Lissabon.

\section{4.-25. Januar 2013, Lissabon}

\section{Eröffnung und Begrüßung}

Dr. Artur Santos SILVA, Präsident der Fundação Calouste Gulbenkian, Lissabon

S.E. Helmut ELFENKÄMPER, Botschafter der Bundesrepublik Deutschland in Portugal, Lissabon Prof. Dr. Nuno Severiano TEIXEIRA, Direktor, Instituto Português de Relações Internacionais da Universidade Nova de Lisboa (IPRI-UNL), Lissabon

Prof. Dr. Mathias JOPP, Direktor, Institut für Europäische Politik (IEP), Berlin

\section{Ansprachen der Außenminister}

Dr. Paulo PORTAS, Außenminister der Portugiesischen Republik, Lissabon

Dr. Guido WESTERWELLE, MdB, Bundesminister des Auswärtigen, Berlin

Portugal und Deutschland in der Wirtschaftsund Währungsunion: Krisenmanagement und Solidarität

Petra MERKEL, MdB, Vorsitzende des Haushaltsausschusses des Deutschen Bundestags, Berlin

* Ann-Sophie Gast, Institut für Europäische Politik, Berlin. 
Einen größeren Bogen spannend, griff das Forum auch Themen wie globale Herausforderungen durch das Aufstreben neuer Mächte und die wachsende Bedeutung des transatlantischen und des transpazifischen Raums auf. Auf deutscher wie portugiesischer Seite wurde das erste Deutsch-Portugiesische Forum als wichtiger Beitrag zu einem besseren Verständnis und intensiveren Beziehungen der beiden Staaten aufgefasst. Das Forum soll 2014 mit der zweiten Jahrestagung in Berlin fortgesetzt werden.

\section{Eine starke Partnerschaft in Zeiten der Krise}

Deutschland und Portugal pflegen seit Langem gute bilaterale Beziehungen. Deutschland förderte den Aufbau demokratischer Strukturen nach der Nelkenrevolution 1974 und unterstützte 1985/86 aktiv den Beitritt Portugals zur Europäischen Gemeinschaft. Die wirtschaftlichen Beziehungen sind eng; Deutschland ist Portugals zweitwichtigster Handelspartner. In den letzten Jahren haben sich die politischen und gesellschaftlichen Kontakte zwischen beiden Staaten intensiviert. Bundeskanzlerin Angela Merkel besuchte Portugal zuletzt im November 2012.

Auch der deutsche Außenminister Guido Westerwelle nutzte das Deutsch-Portugiesische Forum, um die Bedeutung der Beziehungen zu Portugal zu unterstreichen. Er mahnte an, dass sich die Europäische Union neu aufstellen müsse, um auf globaler Ebene mithalten zu können. Auch internationale Organisationen wie die Vereinten Nationen spiegelten nicht mehr die aktuelle weltpolitische Wirklichkeit wider: Latein- und Südamerika sowie Afrika seien im Sicherheitsrat nicht vertreten, Asien habe nur eine Stimme. Die globalen Kraftzentren hätten sich verschoben und mit dem wirtschaftlichen Aufstieg neuer Mächte wie Brasilien, Indonesien, Indien, Südafrika gehe der Wunsch nach mehr politischer Teilhabe einher.

Vor dem Hintergrund der Finanz- und Schuldenkrise diene das Forum nicht nur als Platt-
Prof. Dr. Paulo MOTA PINTO, Vorsitzender des Europaausschusses der Versammlung der Republik, Lissabon

Steffen KAMPETER, MdB, Parlamentarischer Staatssekretär, Bundesministerium der Finanzen, Berlin

Prof. Dr. Luís CAMPOS E CUNHA, Universidade Nova de Lisboa, Lissabon

Prof. Dr. Ansgar BELKE, Universität DuisburgEssen

Moderation: Prof. Dr. Nuno Severiano TEIXEIRA, Direktor, IPRI-UNL, Lissabon, und Prof. Dr. Mathias JOPP, Direktor, IEP, Berlin

Die neue transpazifische Ära und die

Herausforderung der aufstrebenden Mächte:

Nationale und europäische Antworten auf den globalen Wandel

S.E. João VALE DE ALMEIDA, Botschafter, Leiter der Delegation der Europäischen Union in den Vereinigten Staaten von Amerika, Washington

Dr. Wolf-Ruthart BORN, Staatssekretär a.D., Berlin

Dr. Teresa DE SOUSA, Público, Lissabon

Prof. Dr. Ludger KÜHNHARDT, Zentrum für Europäische Integrationsforschung (ZEI), Universität Bonn

Dr. Patrícia DAEHNHARDT, Universität Lusíada und IPRI-UNL, Lissabon

Moderation: Dr. Teresa PATRICIO GOUVEIA, Fundação Calouste Gulbenkian, Lissabon, und Prof. Dr. Gisela MÜLLER-BRANDECK-BOCQUET, Universität Würzburg

Portugal und Deutschland: Partner auf dem Weg zu ökonomischer Innovation und besserer Wettbewerbsfähigkeit in einer globalisierten Welt

Dr. Vitor BENTO, Staatsrat, CEO Sociedade Interbancária (SiBS) S.A., Lissabon

Prof. Dr. Christian DREGER, Deutsches Institut für Wirtschaftsforschung (DIW), Berlin

Dr. Vitalino CANAS, Mitglied des Ausschusses für Europäische Angelegenheiten der Versammlung der Republik, Lissabon 
form des Dialogs und der Beratung, vielmehr solle dem Partner Portugal die volle Unterstützung und der Respekt vor den portugiesischen Reformanstrengungen gezeigt werden. Westerwelle verwies darauf, dass Portugals jüngste Rückkehr an die Finanzmärkte bewiesen habe, dass der Staat auf einem guten Weg sei. In Zeiten der Krise, die nicht nur den Finanzsektor und die Wirtschaft beträfe, sondern die Europäische Union ideell und institutionell grundlegend herausfordere, seien der Dialog zwischen den EU-Mitgliedstaaten sowie enge bilaterale Beziehungen von besonderer Bedeutung. Westerwelle bekräftigte in diesem $\mathrm{Zu}-$ sammenhang die Bereitschaft Deutschlands, Portugal bei der Stärkung des dualen Ausbildungssystems zur Seite zu stehen.

Die aktuelle Krise sei eine Bewährungsprobe für das europäische Lebensmodell, von der die Europäer lernen müssten. Kurzfristige Krisenmaßnahmen seien wichtig, aber es gelte vor allem, die Debatte über die Lehren aus der Krise anzustoßen und über langfristige Lösungen und Reformen nachzudenken. Gleichzeitig müssten die Europäer wieder empfänglicher für gute Nachrichten werden, denn die Anpassungsmaßnahmen in den Programmstaaten zeigten langsam Wirkung. Es gelte nicht zu vergessen, dass Haushaltskürzungen gerade die Menschen mit niedrigem Einkommen besonders hart träfen. Weiterhin betonte er, dass die deutsche Politik nicht allein auf Sparmaßnahmen setze: Solidarität, Haushaltskonsolidierung und Wachstum müssten Hand in Hand gehen, um das Vertrauen in Europa wieder zu stärken. Letztendlich könnten die Mitgliedstaaten gestärkt aus der Krise hervorgehen. Europa sei eine Schicksalsgemeinschaft und die Europäer müssten noch lange zusammenarbeiten, denn die Europäische Union sei ihre Wohlstands- und Lebensversicherung.

Der portugiesische Außenminister Paulo Portas hob hervor, dass die deutsch-portugiesische Partnerschaft keine einseitige Partnerschaft ist. Enge wirtschaftliche Verflechtungen und wechselseitige Abhängigkeiten könnten zu einer Win-win-Situation für beide Staaten füh-
Prof. Dr.-Ing. Dirk ELIAS, Präsident, Fraunhofer AICOS Portugal, Porto

Dr. António MELO PIRES, CEO, Volkswagen Autoeuropa, Palmela

Dr. Carlos MELO RIBEIRO, CEO, Siemens S.A., Amadora

Moderation: S.E. Luís DE ALMEIDA SAMPAIO, Botschafter der Portugiesischen Republik in Deutschland, Berlin, und Prof. Dr. Wim KÖSTERS, Rheinisch-Westfälisches Institut für Wirtschaftsforschung (RWI), Essen

\section{Die Zukunft Europas}

Jo LEINEN, MdEP, Präsident der Internationalen Europäischen Bewegung, Brüssel

S.E. Domingos FEZAS VITAL, Ständiger Vertreter von Portugal bei der Europäischen Union, Brüssel

Karlfried BERGNER, Referatsleiter E 09, Auswärtiges Amt, Berlin

Dr. José PENA DO AMARAL, Banco Português de Investimento (BPI), Lissabon

Prof. Dr. Gabriele ABELS, Universität Tübingen

Prof. Dr. António GOUCHA SOARES, Instituto Superior de Economia e Gestão (ISEG), Lissabon

Peter M. SCHMIDHUBER, Ehemaliges Mitglied der Europäischen Kommission; ehemaliges Mitglied des Direktoriums der Deutschen Bundesbank, München

Moderation: Prof. Dr. Maria João RODRIGUES, Beraterin der Europäischen Kommission, Brüssel/ Lissabon, und Prof. Dr. Martin NETTESHEIM, Universität Tübingen

\section{Abschlussansprachen}

Dr. António VITORINO, Präsident, Notre Europe, Paris

Prof. Dr. Werner WEIDENFELD, Universität München

Vorsitz: Prof. Dr. José GOMES CANOTILHO, Fundação Calouste Gulbenkian, Lissabon

\section{Schlussworte}

Dr. Artur SANTOS SILVA, Präsident, Fundação Calouste Gulbenkian, Lissabon

Prof. Dr. Mathias JOPP, Direktor, IEP, Berlin

Prof. Dr. Nuno Severiano TEIXEIRA, Direktor, IPRI-UNL, Lissabon 
ren. Portugal zeichne sich in der Europäischen Union durch große Integrationsfreundlichkeit und die Bereitschaft aus, Reformen im Land, aber auch in der Europäischen Union zu unterstützen. Zudem stimme Portugal mit Deutschland in vielen Fragen der internationalen und Europapolitik überein. Die gute Partnerschaft zwischen Deutschland und Portugal habe sich auch im Rahmen der ,Gruppe zur Zukunft Europas" der elf Außenminister zur Reform der Europäischen Union gezeigt (der beide Außenminister angehörten). Auch bei den Vereinten Nationen arbeiteten Deutschland und Portugal eng und effektiv zusammen. Portas bezeichnete die Europäische Union trotz der Krise als ein Erfolgsprojekt, das sich nicht nur über den Binnenmarkt und die wirtschaftliche Stärke definiere. Europa sei vor allem ein Raum des Friedens, ein ökonomisch inkludierendes Projekt und eine normative Macht mit globalem Einfluss. Es sei aber auch ein Projekt der Verantwortlichkeit, für das es sich zu kämpfen lohne. Die europäische Vision der sozialen Marktwirtschaft bezeichnete er als einzigartig und schützenswert. Da Europa kein Eliten-, sondern ein Bürgerprojekt sei, bedeute ein Mehr an Europa vor allem ein bürgernäheres Europa.

Vor dem Hintergrund der Schuldenkrise warnte Portas eindringlich vor einer Nord-SüdSpaltung Europas, die dem europäischen Friedensprojekt gefährliche Schäden zufügen könnte. Vorurteile kultureller Art wie zum Beispiel das der „faulen Südländer“ müssten bekämpft werden. Dasselbe gelte aber auch für das weit verbreitete Klischee vom deutschen Spardiktat, das keine Rücksicht auf die Bevölkerung nehme und keinen Blick für Wachstum habe. Solidarität und Zusammenhalt seien die besten Rezepte, um den aktuellen Herausforderungen zu begegnen und die Bevölkerung von der Notwendigkeit der Reformen zu überzeugen.

\section{Krisenmanagement und Solidarität}

Petra Merkel verwies auf die zwar zeitlich versetzt durchgeführten, aber in Deutschland und
Portugal inhaltlich ähnlich gelagerten Strukturreformen. Deutschland habe sie schon durchlaufen, Portugal stünden sie zu großen Teilen noch bevor. Der Grund für die schnelle Regeneration Deutschlands nach der Krise 2008/2009 sei die Agenda 2010, die ab 2003 enorme Reformanstrengungen bedeutet habe und mit Widerstand einhergegangen sei. Solche Reformprozesse seien schwierig, müssten aber angegangen werden. Nur mit soliden Haushalten seien konjunkturpolitische Programme möglich. Merkel betonte in diesem Zusammenhang die Notwendigkeit eines sozialen Dialogs gerade in Zeiten der Krisenpolitik. Die Einbindung der Gesellschaft, von Parteien, Gewerkschaften und Verbänden, bei der Vorbereitung und Durchführung von Anpassungsmaßnahmen sei sehr wichtig. Die parlamentarische Beteiligung in den Geber- wie den Empfängerstaaten von Hilfskrediten sei zentral. Sie warb deshalb nachdrücklich um Verständnis, dass der Deutsche Bundestag sein Budgetrecht gerade bei Krisenmaßnahmen sehr ernst nehme.

Paulo Mota Pinto sprach sich grundsätzlich für eine Bankenunion aus. Was aber bei den Reform- und Sparanstrengungen nicht vergessen werden dürfe, ist, dass nicht alle EU-Mitgliedstaaten in denselben Topf geworfen werden können. Die Staatsschulden hätten unterschiedliche Ursachen, weshalb es wichtig sei, massgeschneiderte Lösungen zu finden. Dies gelte auch für Portugal. Ein weiterer Schwerpunkt der Reformpolitik müsse der Bereich Forschung und Entwicklung sein. Nur so könne die Europäische Union auf dem Weltmarkt bestehen. Voraussetzung dafür sei die Verbesserung der Bildung. Mota Pinto unterstrich, dass Portugal insbesondere vom dualen Ausbildungssystem in Deutschland lernen könne. Portugal müsse ein Reformpaket wie die Agenda 2010 ausarbeiten, das unter anderem eine Reduktion der Staatsverschuldung, die Stärkung des Mittelstandes und die Reform des Ausbildungssystems vorsehe. Abschließend betonte er, dass man ,den Süden' nicht in eine Schublade stecken dürfe. 
Steffen Kampeter begrüßte diese Vorschläge und die von der portugiesischen Regierung ergriffenen Maßnahmen. Er warnte vor zu viel Pessimismus und wies daraufhin, dass Portugal an die Finanzmärkte zurückgekehrt sei, was ein erstes sichtbares Ergebnis der radikalen Sparmaßnahmen sei. Das portugiesische Beispiel zeige aber auch, dass Politik einen langen Atem brauche und sich in Zeiten der Krise nicht an den nächsten Wahlen orientieren dürfe. Er betonte, dass Investitionen für den wirtschaftlichen Aufschwung unentbehrlich sind. Es müsse aber klar sein, wofür und mit welchem Ziel sie eingesetzt würden. Er fügte hin$\mathrm{zu}$, dass solche Investitionen unter anderem von der Europäischen Investitionsbank oder über die Strukturfonds finanziert werden könnten. Auch eine Reform der Unternehmenssteuer sei wünschenswert, um europäische Steuergerechtigkeit herzustellen. 2013 müsse der Reformkurs und die Haushaltskonsolidierung weitergeführt werden, um die Wettbewerbsfähigkeit in den krisengebeutelten EU-Mitgliedstaaten wieder herzustellen. Nur Wettbewerbsfähigkeit und eine solide Haushaltspolitik sicherten stabiles Wachstum.

Luís Campos e Cunha unterstrich, dass man der Krise nicht durch Ausdehnung des Gemeinschaftshaushalts Herr werde, sondern nur durch eine bessere Koordinierung der nationalen Haushaltspolitiken. Dabei müsse die individuelle Situation der Mitgliedstaaten im Blick behalten werden. Eurobonds lösten die bestehenden Probleme nicht, sondern vergemeinschafteten nur die Schulden. Eine solide Haushaltspolitik sei Voraussetzung für das reibungslose Funktionieren der Wirtschaft.

Ansgar Belke erwiderte, dass die unbegrenzten Käufe von Staatsanleihen und die längerfristigen Refinanzierungsgeschäfte der Europäischen Zentralbank ein starkes Zeichen der Solidarität seien und bereits Vertrauen an den Finanzmärkten geschaffen hätten. ,Handelsbilanzdefizite` sollten durch eine Steigerung der Exporte ausgeglichen werden. Eine große Gefahr für das Vertrauen in die Wirtschaft und die Haushaltskonsolidierung sah er in den Ratings.
Missbrauch von Ratings müsse eingedämmt, und Steuerhinterziehung europäisch geahndet werden, um effektiv eingegrenzt zu werden. Andernfalls werde den Mitgliedstaaten ihre finanzielle Basis geraubt. Belke lobte Portugal für die beeindruckende Konsolidierung der Staatsfinanzen, die im Gegensatz zu anderen Eurostaaten ohne die Einsetzung einer Expertenregierung gelungen sei.

Die Beiträge verdeutlichten, dass Deutschland und Portugal an einer Vertiefung der Wirtschafts- und Währungsunion sowie einer besseren Koordinierung der Wirtschafts- und Fiskalpolitiken interessiert sind. Allerdings legten die Diskussionen auch Unstimmigkeiten hinsichtlich des ,besten Rezepts' offen. Insbesondere die portugiesischen Redner betonten, dass Sparmaßnahmen allein die Eurozone nicht konsolidieren können, sondern auch Wachstumsimpulse notwendig seien. In vielen Wortmeldungen wurde auch hervorgehoben, dass die zu tragenden Lasten in der Bevölkerung ungleich verteilt seien.

\section{Globale Herausforderungen}

Globalisierung, permanente Anpassungsprozesse, globale Machtverschiebungen, aufstrebende Mächte mit hohen wirtschaftlichen Wachstumsraten und die in den letzten Jahren deutlich gewordene Orientierung der US-Außenpolitik auf die asiatisch-pazifische Region fordern nicht nur nationale Antworten auf den globalen Wandel, sondern vor allem auch gesamteuropäische Lösungsstrategien, um die Europäische Union zwischen den weltpolitischen Schwergewichten weiterhin nachhaltig positionieren zu können.

Wolf-Ruthart Born, João Vale de Almeida, Teresa de Sousa, Ludger Kühnhardt und Patrícia Daehnhardt als Panellisten waren sich zwar einig, dass auch die Europäische Union einen Anpassungsprozess initiieren müsse, um den Veränderungen auf globaler Ebene gerecht zu werden. Davon sei jedoch nicht allein die europäische Außenpolitik, sondern alle Politikfelder betroffen. Während einige der Sprecher 
auf die bereits erreichten Fortschritte, insbesondere die Schaffung des Europäischen Auswärtigen Dienstes (EAD), verwiesen, betonten andere, dass die Europäische Union zuerst ihre Rolle im globalen Umfeld definieren müsse, bevor weitere Reformen unternommen werden könnten. Angesichts des zunehmenden Engagements der USA im pazifischen Raum wurde die Verantwortung der Europäer unterstrichen, auf dem eigenen Kontinent und in ihrer Nachbarschaft, einschließlich des Nahen Ostens, für Frieden und Sicherheit zu sorgen. Dennoch dürfe die Wahrnehmung der eigenen Verantwortung nicht zu einer Abwendung von der großen internationalen Politik führen. Von besonderer Wichtigkeit seien die Beziehungen zu Asien und Afrika sowie eine gestärkte transatlantische Partnerschaft, die auch Südamerika einbeziehe.

Wirtschaftliche Innovation und Wettbewerbsfähigkeit als Antwort auf die Krise?

Globalisierung unterwirft Forschung und Entwicklung einem fortwährenden Transformationsprozess und schafft einen permanenten Anpassungsdruck für Märkte weltweit. Insbesondere die derzeitige Krise zwingt die Mitgliedstaaten der Europäischen Union, ihre internationale Wettbewerbsfähigkeit auszubauen und wirtschaftliche Innovationen voranzutreiben, um im globalen Wettbewerb bestehen zu können.

Luis de Almeida Sampaio unterstrich die einzigartige geostrategische Lage Portugals, die viel Potenzial für die deutsch-portugiesischen Beziehungen böte. Portugal spiele eine wichtige Rolle in der $\mathrm{NATO}^{1}$ und pflege enge Kontakte mit aufstrebenden Staaten wie Brasilien oder Angola. Um die wissenschaftliche und technologische Vernetzung zwischen Portugal und Deutschland weiter auszubauen, böte das Forum eine gute Grundlage. Wim Kösters unterstrich, dass sich das für Europa notwendige Wachstum nur über mehr Innovation, Wettbewerbsfähigkeit und einen „Wettbewerb der
Vielfalt" erreichen lasse. Er habe aufgrund früherer Erfahrungen Zweifel an Forderungen nach ,mehr Europa', wenn sie auf rein zentralistische Lösungen hinausliefen.

Es wurde die Frage thematisiert, wieso Portugal von der Schuldenkrise so hart getroffen wurde und wie in der Zukunft Portugals Krisenanfälligkeit reduziert werden könne. Laut Vitor Bento gehört Portugal zu den Verlierern der EU-Erweiterung. Während es zu Beginn seiner Mitgliedschaft eine erhebliche Wohlstandssteigerung erfuhr, sei es durch die Verschiebung der europäischen Außengrenzen zur europäischen Peripherie geworden, was nicht nur wirtschaftliche, sondern auch psychologische Folgen habe. Portugal müsse seine Rolle in der Europäischen Union neu definieren, sein atlantisches Potenzial nutzen und seine Beziehungen zu wichtigen Drittstaaten intensivieren.

Auch Christian Dreger stimmte der These zu, dass Portugal zu den Verlierern der Erweiterung gehöre, begründete dies aber mit dem enorm gestiegenen Konkurrenzdruck innerhalb der Europäischen Union. Die Krise habe strukturelle Defizite in Portugal aufgedeckt, wie zum Beispiel die hohe Auslandsverschuldung, die gestiegenen Lohnstückkosten, die Wachstumsschwäche der Industrie- und Gütermärkte. Um diese Defizite zu beheben und Portugals Wettbewerbsfähigkeit wieder zu steigern, müssten nun dringend die öffentlichen Haushalte konsolidiert, der Kündigungsschutz gelockert und die Märkte strukturell reformiert werden. Die Lohnstückkosten müssten nicht nur durch die Senkung der Nominallöhne, sondern auch durch eine Produktivitätssteigerung abgebaut werden. Schließlich dürfe Portugal nicht allein auf exportinduziertes Wachstum setzen, sondern müsste seinen Fokus auf Technologie und Innovation verschieben.

António Melo Pires nahm eine drastischere Position ein und gab Portugal die volle Schuld an

1 North Atlantic Treaty Organization. 
der derzeitigen Lage. Es habe unter anderem die europäischen Strukturfonds nicht richtig genutzt, um seine Wettbewerbsfähigkeit zu stärken, und könne deshalb heute im europäischen und globalen Wettbewerb nicht mehr mithalten. Außerdem führten Defizite im Bildungssystem zu einem Mangel an mittleren Führungskräften für die Industrie. Die Hochschulbildung in Portugal sei zwar exzellent, aber leider „sitze sie im Elfenbeinturm“ und generiere keine anwendungsorientierten Forschungsergebnisse. Auch Dirk Elias bestätigte, dass das portugiesische Bildungssystem insbesondere im universitären Bereich sehr gut sei. Portugal müsse das vorhandene Potenzial nur besser nutzen. Er sehe den Staat in der Pflicht, das unternehmerische Denken zu fördern und die Wissenschaft stärker mit der Wirtschaft zu vernetzen. Carlos Melo Ribeiro regte unter anderem an, den Tourismus zwischen Portugal und Deutschland weiter zu fördern. Bisher kämen zu wenige deutsche Touristen nach Portugal. Außerdem sei Portugal im Hinblick auf den Mangel an Ingenieuren in Deutschland ein interessanter Partner.

Auf portugiesischer Seite wurden aber auch Differenzen und Schwierigkeiten in den deutsch-portugiesischen Beziehungen thematisiert. Vitalino Canas erklärte, dass die Sozialistische Partei nicht immer mit der deutschen Politik übereinstimme. Der deutsche Widerstand gegen eine europäische Wachstumsagenda schade ganz Europa. Auch befürchtete er, dass in Deutschland lediglich die politische Elite ein positives Portugalbild habe, die Mehrheit der Bürger aber den Vorurteilen mangelnder Disziplin der ,Südländer' anhinge. Trotz aller Kritik wünschte sich Canas ein starkes Deutschland in Europa, das seine Führungsrolle verantwortungsbewusst wahrnehme.

Die Panellisten waren sich einig, dass die Krise strukturelle Defizite in der Eurozone aufgedeckt habe. Portugal müsse unter anderem seine Auslandsverschuldung und seine Lohnstückkosten senken, die Industrie stärken, die öffentlichen Haushalte konsolidieren und die Güter- und Arbeitsmärkte reformieren. Die portugiesischen Teilnehmer wünschten sich ferner, eine wachstumsorientiert Politik und dass die Bundesregierung Investitionen in Portugal aktiv fördere.

\section{Die Zukunft der Europäischen Union}

Seit knapp vier Jahren hält die Staatsschuldenkrise die Tagespolitik der Europäischen Union schon in Atem. Dabei wurden nicht nur Konstruktionsfehler der Wirtschafts- und Währungsunion deutlich, die es zu beheben gilt, sondern auch, dass die Union insgesamt handlungsfähiger werden muss, um den Herausforderungen des 21. Jahrhunderts begegnen zu können.

Maria João Rodrigues und Martin Nettesheim erinnerten an die großen Herausforderungen, vor denen die Europäische Union derzeit stehe, die nur durch eine legitimierte Fortentwicklung der europäischen Institutionen und mehr Vertrauen der Bürger in diese gemeistert werden könnten. Außerdem warfen sie die schwierige Frage auf, wie die Europäische Union künftig ein Gleichgewicht zwischen Solidarität und Verantwortung herstellen könne.

Die Panellisten stimmten überein, dass erneuter Reformbedarf in der Europäischen Union bestehe. Dieser müsse, so Jo Leinen in naher Zukunft durch eine weitere Reformrunde aufgearbeitet werden, um die Legitimität des Integrationsprojekts wieder zu stärken. Leinen bezeichnete die sogenannte Bloomberg-Rede des britischen Premierministers David Cameron von Januar 2013 als „Frontalangriff“ auf die Institutionen der Europäischen Union. Die Rede böte zwar auch Anlass zur Debatte über die gemeinsame Vision von Europa. Ein „Europa à la carte" dürfe es allerdings nicht geben.

Als zentrale Reformaufgaben wurden die Stärkung des Europäischen Parlaments unter anderem durch ein eigenes Initiativrecht, die Schaffung einer einheitlichen transparenten Exekutive und eine Intensivierung der Kooperation zwischen dem Europäischen und den nationalen Parlamenten genannt. Die Stärkung 
der sozialen Dimension und der Solidarität zwischen den Mitgliedstaaten seien weitere zentrale Aufgaben, die zu einer besseren Legitimation der Europäischen Union beitrügen. Schließlich müsse die Gemeinsame Außenund Sicherheitspolitik fortentwickelt werden, damit Europa zukünftig als politische Einheit auf der Weltbühne wahrgenommen werde und nicht in der Bedeutungslosigkeit verschwände.

Zwei Entwicklungen im aktuellen Krisenmanagement trügen zur Schwächung der Legitimität der Europäischen Union bei: Aus der Perspektive kleiner und mittlerer Mitgliedstaaten sei die dominante Rolle Deutschlands unbefriedigend und werde von Politikern und Bürgern kritisiert. José Pena do Amaral sprach in diesem Kontext von einem ,unipolaren Moment", in dem keine Entscheidung ohne, aber auch keine gegen Deutschland möglich sei. Weiterhin werde der Ansatz, die Reform der Europäischen Union außerhalb des Vertragswerks voranzutreiben, als ein Bruch des Primärrechts wahrgenommen und stelle die Europäische Union als Rechtsgemeinschaft infrage. Dieser Weg sei als Notlösung in der Krise noch akzeptabel, dürfe aber nicht Schule machen.

Domingos Fezas Vital warnte, dass der $\mathrm{Zu}-$ sammenbruch des Euros die Europäische Union als Ganzes gefährden könne. Peter Schmidhuber wies auf den Wiederspruch hin, dass einerseits eine handlungsfähige Wirtschafts- und Währungsunion verlangt werde, andererseits aber an der Souveränität der Mitgliedstaaten festgehalten werde. Die Eurokrise habe zudem gezeigt, dass heute ein Europa der zwei Geschwindigkeiten existiere. Dieser Trend werde noch durch die Erweiterungspolitik verstärkt, die zu immer mehr Variationen und einem größeren Wohlstandsgefälle zwischen den Mitgliedstaaten führe und sowohl die wirtschaftliche als auch die soziale Kohärenz der Europäischen Union gefährde.

Pena do Amaral warnte vor einem Bruch zwischen den Eurostaaten und den Staaten ohne Gemeinschaftswährung. António Goucha Soa- res wies darauf hin, dass die Krise zu einer Intergouvernmentalisierung der EU-Politik und der Institutionen geführt habe. Diese Entwicklung müsse durch eine Vertiefung der Integration wieder rückgängig gemacht werden. $G a$ briele Abels betonte, dass die derzeitige Krise vor allem auch eine Vertrauenskrise sei, die nur durch eine Demokratisierung und die Widerherstellung des sozialen Friedens durch einen neuen Gesellschaftsvertrag zu beheben sei. In der Diskussion über die Weiterentwicklung der Union schlug Karlfried Bergner vor, nach der kommenden Wahl zum Europäischen Parlament über einen neuen Konvent nachzudenken. Jetzt komme der Zeitpunkt, langfristig Lehren aus der Krise zu ziehen und die Europäische Union zu reformieren. In diesem $\mathrm{Zu}$ sammenhang sei es wichtig, dass sich die Europäische Union nicht ausschließlich auf sich selbst konzentriere, sondern auch auf globaler Ebene versuche, ihre Handlungsfähigkeit zu vergrössern.

Die Panellisten waren sich einig, dass weder differenzierte Integration noch eine Desintegration eine Lösung der gegenwärtigen Probleme Europas darstellen. Europa müsse den Bürgern näher gebracht werden, mit dem Ziel, dass die Menschen sich wieder für die Europäische Union interessierten und an den Wahlen zum Europäischen Parlament teilnähmen. Die Europäische Union dürfe in den Krisenstaaten nicht nur für harte Sparpolitik, sondern müsse wieder für ein inkludierendes soziales Friedensprojekt stehen.

\section{Positive Bilanz}

Das Deutsch-Portugiesische Forum endete mit den Reden von António Vitorino und Werner Weidenfeld. Letzterer unterstrich in seiner Rede die Bedeutung von Narrativen, um die Europäische Union zu legitimieren. In der Vergangenheit hätten zwei Narrative genügt, um die Bedeutung der europäischen Integration zu versinnbildlichen. Das erste Narrativ beschrieb Europa als Friedensprojekt und Alternative zu den beiden Weltkriegen, das zweite als Freiheitsprojekt und Alternative zu den sozialisti- 
schen Staaten während des Kalten Kriegs. Beide Narrative, die gleichzeitig zur Legitimation der europäischen Integration dienten, genügten aber nicht mehr, um die Bürger von der Europäischen Union zu überzeugen. Europa brauche neue Wahrnehmungsmuster und Begründungszusammenhänge. Heute spiele insbesondere das Narrativ „Europa als globaler Akteur“ eine wichtige Rolle. Die Akzeptanz, eine (zivile) Weltmacht zu sein, genieße die Europäische Union bereits. Nun gehe es darum, diesen Status mit Leben zu füllen. Wenn die gegenwärtige Krise überwunden und das Institutionensystem der Europäischen Union reformiert sind, werde die Außenpolitik das „Megathema“, prognostizierte Weidenfeld. Vitorino nutzte seine Rede, um die Bedeutung freundschaftlicher und solidarischer Beziehungen zwischen den Mitgliedstaaten der Europäischen Union insbesondere in Zeiten der Krise hervorzuheben. Hierzu habe das Forum einen wichtigen Beitrag geleistet.

Insgesamt unterstrichen alle Teilnehmer den Wert der zweitägigen Konferenz und sprachen sich für die Institutionalisierung des DeutschPortugiesischen Forums aus. Die portugiesische Presse berichtete umfassend über die Ver- anstaltung und gab ein durchweg positives Echo. Insbesondere die Anerkennung der portugiesischen Reformanstrengungen und die Würdigung der Rückkehr Portugals an die Finanzmärkte durch hochrangige deutsche Politiker fanden Erwähnung. Auch wenn eine zweitätige Konferenz nur einen Anstoß für die Stärkung der deutsch-portugiesischen Beziehungen geben kann, so konnte das Forum doch $\mathrm{zu}$ einem besseren gegenseitigen Verständnis und zur Stärkung der Solidarität zwischen Deutschland und Portugal beitragen. Besonderer Dank für die ausgezeichnete Zusammenarbeit gilt dem Instituto Português de Relações Internacionais (IPRI-UNL), der Fundação Calouste Gulbenkian sowie der Botschaft der Bundesrepublik Deutschland in Portugal, der portugiesischen Nachrichtenagentur Lusa und dem Goethe-Institut in Lissabon für die vielfältige Hilfe und Unterstützung bei der Vorbereitung des Forums. Ganz besonderer Dank gilt auch dem Auswärtigen Amt für die Förderung des Forums, ohne die seine Durchführung nicht möglich gewesen wäre. Die zweite Jahrestagung des Deutsch-Portugiesischen Forums wird 2014 in Berlin stattfinden. 